Trauma Surgery \& Acute Care Open

Department of Surgery, SUNY Upstate Medical University, Syracuse, New York, USA

Correspondence to Dr Robert N Cooney, Department of Surgery, SUNY Upstate Medical University, 750 E. Adams St, Suite 8141 Syracuse, NY 13210, USA; Cooneyr@upstate.edu

Received 7 November 2017 Accepted 9 November 2017

\section{Spontaneous splenic rupture due to marginal zone lymphoma}

\author{
Natesh Yepuri, Napat Pruekprasert, Taewan Kim, Robert N Cooney
}

An 81-year-old man collapsed at home after complaining of left upper quadrant (LUQ) abdominal pain and presented to the local hospital. $\mathrm{He}$ was hypotensive on arrival, but stabilized with transfusion of 2 units of packed red blood cells. His abdominal examination was significant for LUQ tenderness with no signs of peritonitis. Abdominal CT scan (figure 1) revealed splenomegaly with grade 3 splenic injury and moderate hemoperitoneum. Blood work was significant for anemia and elevated creatinine $(\mathrm{Cr} 1.9 \mathrm{mg} / \mathrm{dL})$. Spontaneous splenic rupture (SRS) was suspected and the patient was transferred to the nearest level 1 trauma center for management.

\section{WHAT WOULD YOU DO?}

A. Non-operative management (NOM).
B. Emergency splenectomy.

C. Splenic angioembolization (SAE).

D. Stabilization and laparoscopic total splenectomy.

\section{WHAT WE DID AND WHY}

Correct answer D

We stabilized the patient and performed a laparoscopic total splenectomy. The patient's vital signs were normal on arrival, so he was monitored in the intensive care unit with plan for emergency splenectomy if he destabilized. His creatinine and hematocrit normalized, so he underwent laparoscopic hand-assisted splenectomy. Operative findings revealed splenomegaly with hemoperitoneum. The spleen was mobilized from its attachments, the splenic vessels were stapled and the spleen was removed. Histopathological examination

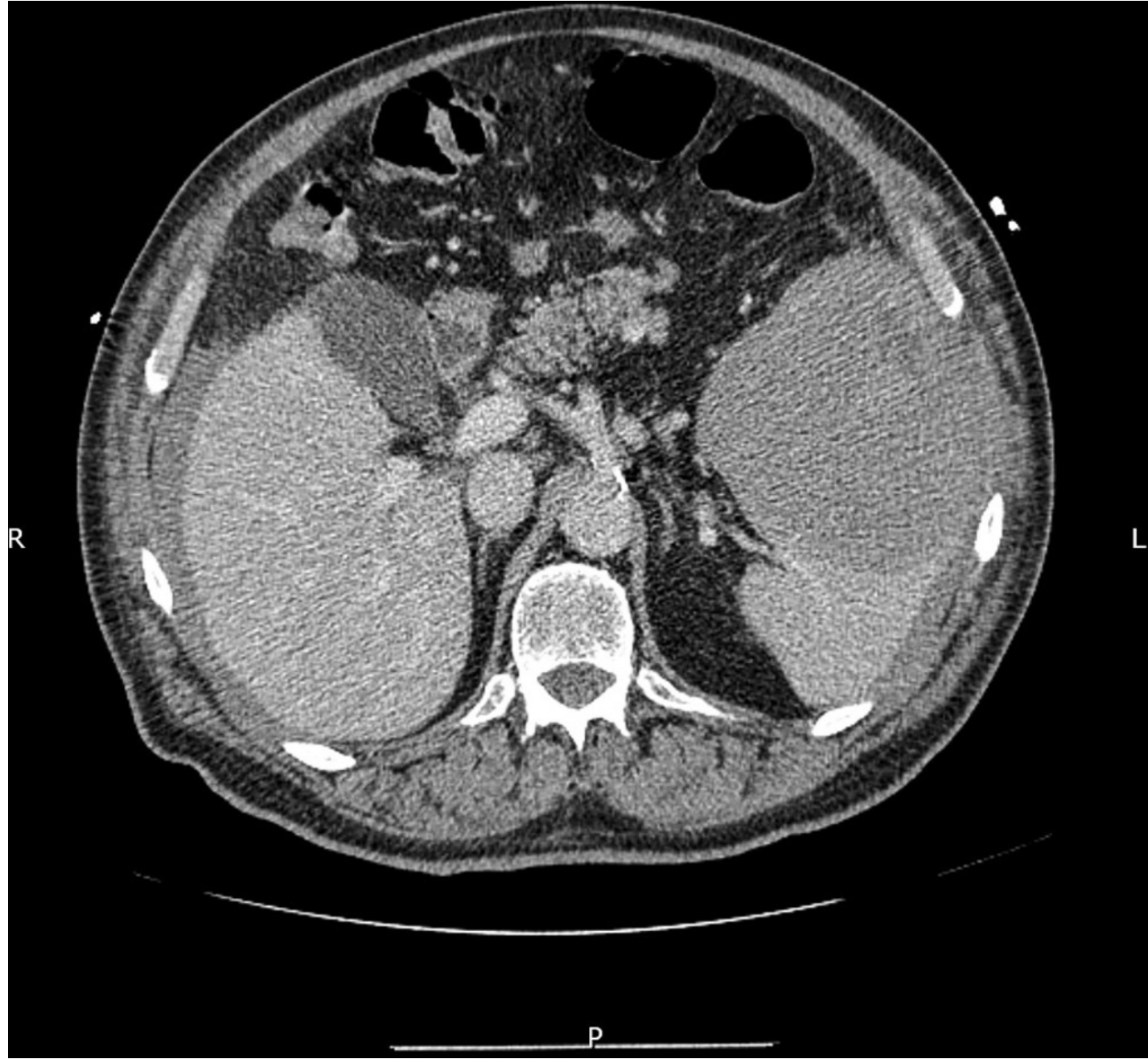

Figure 1 Abdominal CT depicting splenic rupture.
Pruekprasert N, Kim T, et al. Trauma Surg Acute Care Open 2017:2:1-2. 
revealed marginal zone lymphoma. His postoperative course was uneventful. He received postsplenectomy vaccinations and is being followed by hematology for his lymphoma.

Atraumatic SRS is an uncommon condition which occurs unexpectedly and may require emergency splenectomy to prevent exsanguination. The diagnosis may be missed or delayed because of low clinical suspicion, especially in the absence of trauma. Many patients with SRS have underlying splenic pathology including associated infections, inflammatory conditions or neoplasm. Although the spleen is often involved in hematological malignancies, splenic rupture is an infrequent occurrence until the infiltrating malignant cells cause architectural changes, parenchymal fragility and vascular occlusion resulting in thrombosis with infarction thereby exceeding the capacity of the non-distensible splenic capsule causing rupture. Common symptoms and signs of SRS include abdominal pain, tenderness and guarding, hypotension, dizziness and syncope, although the diagnosis is hard to make on clinical findings alone.

Although this patient was hemodynamically normal after his initial transfusion, NOM is risky because the diseased, injured spleen is unlikely to heal normally and NOM is associated with failure in the setting of splenic pathology. Additionally, increased patient age is a relative contraindication to NOM and is associated with failure of NOM. If the patient's vital signs and hematocrit had not normalized after his initial blood transfusion and there were signs of ongoing bleeding, emergency splenectomy would be indicated. Patients with SRS of malignant etiology should generally undergo immediate total splenectomy. However, in this case, the patient had not yet been diagnosed with underlying splenic pathology.

Splenic angioembolization (SAE) is commonly performed in hemodynamically normal patients with high-grade splenic injuries and/or active extravasation on CT scan to help stop the bleeding and increase the chances of successful NOM. Although SAE is associated with preservation of splenic function, there are associated risks including infection, infarction, persistent bleeding requiring splenectomy and contrast-induced acute kidney injury (AKI). In this case, the patient's creatinine clearance was decreased due to his age and prehospital hypotension which increase the risk of contrast-induced AKI if SAE were performed.

The patient's age, splenomegaly and SRS were concerning for the presence of associated splenic malignancy which we felt would best be treated by splenectomy for several reasons.
First, histological examination of the spleen would establish the etiology of the SRS and any underlying systemic disease(s). Second, a significant number of malignant diseases may cause SRS and frequently require total splenectomy as treatment. Finally, there is a good chance that splenic function is already compromised due to pathologic alterations or infiltration of the splenic parenchyma, resulting in functional hyposplenism. For these reasons, we allowed his kidney function to recover, then performed laparoscopic splenectomy to facilitate his recovery from surgery.

The pathology report revealed marginal zone lymphoma (MZL) of the spleen. MZL is an indolent, mature B-cell neoplasm with three entities: nodal MZL, splenic MZL, and extranodal MZL of mucosa-associated lymphoid tissue type. Splenic MZL comprises less than $1 \%$ of all lymphomas that affects elderly or middle-aged patients in the sixth decade without gender predominance. Its definitive diagnosis relies on histology characterized by massive splenic involvement with micronodular pattern infiltration and marginal zone differentiation. SRS has rarely been reported as a complication of MZL.

In conclusion, this case emphasizes the importance of considering hematological malignancies when encountering SRS in elderly patients with splenomegaly. A high index of suspicion should be maintained by emergency physicians and surgeons during the evaluation of these patients. Although NOM and SEA are commonly used in patients with blunt splenic trauma, elderly patients with SRS and splenomegaly may benefit from urgent or delayed splenectomy to properly diagnose and treat associated splenic pathology.

Contributors NY, NP and RNC were involved in the writing of the article. NY, NP and RNC conducted literature search. RNC and TK were involved in the surgical management of patient. RNC and TK carried out the critical revision of the article.

Competing interests None declared.

Provenance and peer review Not commissioned; internally peer reviewed.

Open Access This is an Open Access article distributed in accordance with the Creative Commons Attribution Non Commercial (CC BY-NC 4.0) license, which permits others to distribute, remix, adapt, build upon this work non-commercially, and license their derivative works on different terms, provided the original work is properly cited and the use is non-commercial. See: http://creativecommons.org/ licenses/by-nc/4.0/

(c) Article author(s) (or their employer(s) unless otherwise stated in the text of the article) 2017. All rights reserved. No commercial use is permitted unless otherwise expressly granted. 